\title{
Influence of anti-TNF therapy and homocysteine level on carotid intima-media thickness in rheumatoid arthritis patients
}

\author{
DANIELA ANGHEL ${ }^{1,2^{*}}$, CARMEN ADELLA SîRBU ${ }^{2,3}$, ELENA-MĂDĂLINA HOINOIU ${ }^{1}$, \\ OANA-GEORGIANA PETRACHE ${ }^{1}$, CRISTINA-FLORENTINA PLEŞA ${ }^{3,4}$, \\ MARIA MAGDALENA NEGRU ${ }^{5,6^{*}}$ and FLORENTINA IONIŢĂ-RADU ${ }^{6,7}$ \\ ${ }^{1}$ Department of Internal Medicine, Central Military Emergency University Hospital, 010242 Bucharest; \\ ${ }^{2}$ Department of Medico-Surgical and Prophylactic Disciplines, Faculty of Medicine, 'Titu Maiorescu' University, \\ 031593 Bucharest; ${ }^{3}$ Department of Neurology, Central Military Emergency University Hospital, 010242 Bucharest; \\ ${ }^{4}$ Department of Preclinical Disciplines, Faculty of Medicine, 'Titu Maiorescu' University, 031593 Bucharest; \\ ${ }^{5}$ Department of Internal Medicine and Rheumatology, 'Sf. Maria' Clinical Hospital, 011172 Bucharest; \\ ${ }^{6}$ Department of Internal Medicine, 'Carol Davila' University of Medicine and Pharmacy, 020022 Bucharest; \\ ${ }^{7}$ Department of Gastroenterology, Central Military Emergency University Hospital, 010242 Bucharest, Romania
}

Received August 6, 2021; Accepted September 7, 2021

DOI: $10.3892 /$ etm.2021.10981

\begin{abstract}
It is a well-known fact that disruptions in the immune system and systemic inflammation are associated with accelerated atherosclerosis in rheumatoid arthritis (RA) patients. Elevated levels of tumor necrosis factor $\alpha$ (TNF- $\alpha)$, a major pro-inflammatory cytokine, are involved in endothelial cell activation of medium and large arteries, leading to increased endothelial permeability, generation of superoxide anion radical and hydrogen peroxide, and decreased availability of nitric oxide (NO). The present study aims to determine the influence of anti-TNF therapy and homocysteine (Hcy) levels on the carotid intima-media thickness (IMT) in patients with RA. Assessments were performed on 115 patients diagnosed with RA on biological treatment to determine the evolution of IMT and Hcy levels. Carotid ultrasonography was used to assess the IMT, as a fast and easy tool for the prediction of cardiovascular events in patients with RA. The first measurement of IMT was noted as IMT1, followed by a second measurement after 1 year, noted as IMT2. The group of patients was divided into approximately three equal groups, each being treated with a different biological product,
\end{abstract}

Correspondence to: Dr Carmen Adella Sîrbu, Department of Medico-Surgical and Prophylactic Disciplines, Faculty of Medicine, 'Titu Maiorescu' University, 67A Gheorghe Petraşcu Street, 031593 Bucharest, Romania

E-mail: sircar13@yahoo.com

${ }^{*}$ Contributed equally

Key words: anti-TNF, homocysteine, intima-media thickness, rheumatoid arthritis, infliximab, adalimumab, etanercept, cardiovascular risk respectively, etanercept, adalimumab, and infliximab. In the 3 groups, after 1 year of anti-TNF- $\alpha$ therapy, IMT2 progression was significantly reduced compared to baseline. No significant differences were found among the three groups of treatment. A strong association was observed between IMT1-IMT2 in the etanercept group $(\mathrm{P}<0.001, \mathrm{r}=0.758)$, in the adalimumab group $(\mathrm{P}<0.001, \mathrm{r}=0.761)$ and in the infliximab group $(\mathrm{P}<0.001, \mathrm{r}=0.829)$. The low level of Hcy 2 after 12 months of anti-TNF- $\alpha$ therapy was significantly correlated with a decrease in IMT2 $(\mathrm{P}<0.001)$ in patients who had a high level of Hcy and IMT $>0.9 \mathrm{~mm}$ at baseline. The results from the present study showed that biological treatment and the low level of homocysteinemia reduced the cardiovascular risk in RA, regardless of the treatment chosen (infliximab, adalimumab, or etanercept).

\section{Introduction}

Rheumatoid arthritis (RA) is a chronic inflammatory systemic disease predisposing to atherosclerosis and high cardiovascular risk $(1,2)$. As a rule, chronic inflammation has a major role in the pathogenesis of atherosclerosis which is characterized by increased mortality. Many other risk factors, such as dyslipidemia, hypertension, homocysteine (Hcy) levels, obesity, diabetes mellitus, and cigarette smoking are included $(3,4)$. Cardiovascular and cerebrovascular diseases are the leading cause of morbidity and mortality worldwide (5). Patients with RA develop accelerated atherosclerosis and require close monitoring. Their life expectancy is reduced by 5-10 years and $35-50 \%$ of deaths result from cardiovascular disease (6). RA is characterized by a significant biological inflammatory syndrome that leads to a significant risk of atherosclerosis that can be assessed by carotid intima-media thickness (IMT). In our study, we focused on the use of carotid artery intima-medial thickness as a marker for subclinical atherosclerosis. The measurement 
of carotid IMT is a non-invasive test usually made by external ultrasound that can identify atherosclerosis and monitor its evolution. Ultrasound measurement of carotid artery IMT is a widely used method to assess atherosclerosis with a strong prediction of myocardial infarction and cerebrovascular accidents. $(7,8)$. IMT $\geq 1 \mathrm{~mm}$ indicates a high risk of myocardial infarction and cerebrovascular accidents (9). Biological and imaging analysis by Hcy, respectively IMT becomes more accurate for the evolution of subclinical atherosclerosis. Tumor necrosis factor (TNF), a pro-inflammatory cytokine that is secreted by immune cells, plays a role in immunity, homeostasis, inflammation, lipid metabolism, cell proliferation, and programmed cell death (10). It is the first cytokine that appears in case of injuries or stress. Interleukin (IL)-1 and IL-6, other pro-inflammatory mediators, depend on the release of TNF and appear much later. TNF- $\alpha$ controls vascular permeability, allowing inflammatory cells and macromolecules to go to the injured tissue. Thereby, it allows subendothelial accumulation of blood lipids and the formation of atherosclerotic plaques (11).

Atherosclerosis is associated with naive $\mathrm{T}$ cell-T helper 1 cell (Th0-Th1) response and $\mathrm{CD}^{+} \mathrm{T}$ cells are involved in the process of endothelial injury (12). Studies have shown that in $\mathrm{RA}$, the progression of atherosclerosis is correlated with inflammation. In addition, these patients have a plaque phenotype that is more prone to rupture (13). The vascular injuries that are associated with atherosclerotic diseases are characterized by the activation of reactive oxygen species (ROS), formation and release of inflammatory cytokines, decreased bioavailability of nitric oxide (NO), increased endothelial permeability, all of these leading to endothelial dysfunction (14). Hcy is the demethylated metabolite of methionine which is an essential amino acid. B12, folic acid, and B6 vitamins are very important in the metabolism of Hcy. Supplementation of these vitamins decreases Hcy levels and may contribute to stroke prevention (15).

Hcy, at high concentrations, becomes harmful to endothelial cells, with atherosclerotic and prothrombotic effects (2). Several studies suggest that increased levels of Hcy in plasma are associated with higher cardiovascular mortality, cerebrovascular accidents, dementia, and bone fracture, but more likely Hcy is considered a marker and not the cause for these diseases (16-19). The treatment for RA has a double effect: it slows down the progression of the underlying disease, but also the progression of atherosclerosis $(20,21)$. TNF inhibition in RA patients may reduce insulin resistance, C-reactive protein (CRP), and IL-6, therefore decreasing the risk of accelerated atherosclerosis and overall cardiovascular events $(22,23)$. Statins may also have a favorable effect on IMT progression (9). The use of statins can lead to a reduction in LDL-C, the limitation of LDL-C oxidation, the stabilization, and regression of atherosclerotic plaques. This could theoretically be used in RA patients for the prevention of primary atherosclerosis (24).

\section{Patients and methods}

Subjects. We performed a retrospective observational study on 115 patients, 43 males, and 72 females, who were diagnosed with RA (and met the EULAR/ACR2010 criteria for RA) and started treatment with anti-TNF agents (infliximab, adalimumab, and etanercept) (25). Inclusion criteria were: i) age above 18 years; ii) diagnosis of RA (2010 ACR/EULAR criteria); iii) active disease (DAS28 score above 5; iv) arthralgia measured by Visuale Analogue Scale (VAS) above $40 \mathrm{~mm}$; v) non-responder status to two DMARDs, each on maximal dose for at least 12 weeks. Exclusion criteria were: i) patients with diabetes mellitus, and clinical manifestations of atherosclerosis such as peripheral vascular disease, coronary artery disease, and cerebrovascular disease; ii) age group under 18 years; iii) history of smoking or alcoholism; iv) patients who received treatment with interleukin (IL)-6 inhibitors or Janus kinase (JAK) inhibitors; v) patients who received treatment with other disease-modifying anthirheumatic drugs (DMARDs) other than methotrexate or leflunomide; vi) positive viral hepatitis $(B, C)$ and HIV. Our study aimed to observe the evolution of IMT and Hcy levels in RA patients with biological treatment. The study was conducted in one center ('Carol Davila' Central Emergency Military University Hospital, Bucharest, Department of Internal Medicine 2), between January 2019 and February 2020. The group of patients was divided into approximately three equal groups, each being treated with a different biological product, respectively adalimumab $33 \%$, etanercept $36.5 \%$, and infliximab 30.5\%. At the same time as the biological treatment was administered, the patients received treatment with methotrexate or leflunomide. In our study $71(61.73 \%)$ patients received anti-TNF therapy associated with methotrexate (MTX) and 44 (38.26\%) patients received associated leflunomide. The weekly dose of MTX was $10 \mathrm{mg}$ and the dose of leflunomide was $20 \mathrm{mg}$ per day. All patients who had received MTX received $5 \mathrm{mg}$ of folic acid daily except on the day of MTX.

Measurement of carotid artery IMT. Examination of the carotid wall is a fast method that can detect subclinical alterations in the wall structure and predict subsequent cardiovascular and cerebrovascular accidents. B-mode ultrasonography is a sensitive, noninvasive, reproducible, and broadly available method for the detection of early phases of atherosclerosis in RA patients. Patients were examined in the supine position, in a slight hyperextension of the neck with the head rotated $45^{\circ}$ towards the left or right side. Duplex carotid-intima color Doppler ultrasound (LOGIQ P9) was performed by the same examiner. IMT is seen as a double-line pattern that can be visualized by $2 \mathrm{D}$ ultrasonography in a longitudinal view on both walls of the carotid arteries. We assessed the IMT of the common carotid artery (10 $\mathrm{mm}$ before the bulb). Images of the smallest diameter of the artery (end-diastolic diameter) were captured.

The specialist obtained standardized B-mode images and Doppler flow measurements from the right and left carotid arteries. We considered the highest value recorded in a single patient without noting on which side. As stated by current sonographic criteria, we consider normal IMT if the intima-media complex was $\leq 0.9 \mathrm{~mm}$. An IMT $>0.9 \mathrm{~mm}$ was considered suggestive of thickened intima, while $>1.3 \mathrm{~mm}$ was suggestive of atherosclerotic plaque. Carotid artery plaque was excluded. We performed two examinations: one at the beginning of the treatment (IMT1) and a second measurement 1 year later (IMT2). Hyperhomocysteinemia was considered moderate if the 
levels were between 15 and $30 \mu \mathrm{mol} / 1$, intermediate if the levels were 30-100 $\mu \mathrm{mol} / 1$, and severe if they were higher than $100 \mu \mathrm{mol} / 1$. Levels of Hcy below $15 \mu \mathrm{mol} / 1$ were considered normal (26). Erythrocyte sedimentation rate (ESR), CRP, rheumatoid factor (RF), antibodies directed to cyclic citrullinated peptides (anti-CCP), total cholesterol (TC), and low-density lipoprotein (LDL) were measured to quantify cardiovascular risk. Laboratory limits for ESR were $<10 \mathrm{~mm} / \mathrm{h}$, for CRP $<5 \mathrm{mg} / \mathrm{dl}$, for $\mathrm{TC}<200 \mathrm{mg} / \mathrm{dl}$, and for $\mathrm{LDL}<100 \mathrm{mg} / \mathrm{dl}$. We assessed the disease activity in the RA patients using the DAS-28 score (a disease activity score used in RA that assesses 28 joints). A score below 2.6 indicates a controlled disease, between 2.6 and 5.1 a moderately controlled disease, and over 5.1 uncontrolled disease. In addition, we used the DAS28 score to appreciate the response to the anti-TNF treatment. All patients received a dose of $20 \mathrm{mg} /$ day of atorvastatin as statin therapy which can reduce or reverse carotid IMT progression. The majority of the patients had hypercholesterolemia. This study was approved by the Research Ethics Committee of 'Dr. Carol Davila' Central Military Emergency University Hospital Bucharest (no. 470) and all subjects provided written informed consent.

Statistical analysis. Statistical analysis was executed by SPSS software version 11 (SPSS, Inc.). Pearson correlation coefficient value of 0.01 was considered to reflect statistical significance. Normality was tested with the Shapiro-Wilk test. Mean age, Hcy, ESR, CRP, TC, LDL values are reported as mean $\pm \mathrm{SD}$, and sex, RF, and anti-CCP as a percentage. Continuous variables were expressed as mean \pm SD or in quartiles if not otherwise stated. Logarithmic transformations were used for carotid IMT and other variables that were not normally distributed. Transformations were used for carotid IMT values then they were back-transformed for presentation on the original scale. Univariate comparisons connecting risk factors and carotid IMT were explored using Pearson's and Spearman rank correlations for continuous variables and analysis of variance (ANOVA) for categorical variables. Univariate comparisons between categorical variables and the plaque calcification score were made using logistic regression. Two-sided P-values $\leq 0.05$ were interpreted to have statistical significance for all analyses. Comparison between the groups was made by the Student's t-test. Associations between variables were performed by Pearson correlation coefficients. Linear regression analysis was used for the estimation of IMT predictors. Logistic regression analysis was used for the prediction of atheroma and the estimation of odds ratio (OR) at a confidence interval (CI) of $95 \%$.

\section{Results}

The study group included $115 \mathrm{RA}$ patients who were treated with TNF- $\alpha$ inhibitors (adalimumab $40 \mathrm{mg}$ subcutaneously every two weeks; etanercept $50 \mathrm{mg}$ subcutaneously once a week; and infliximab $3 \mathrm{mg} / \mathrm{kg}$ body intravenously $0,2,6$ weeks and every 8 weeks).

It can be seen that the profile of our typical patient in this cohort was a woman (62.6\%), with seropositive RA, who met the criteria for initiating biological treatment (significant inflammatory syndrome, non-responder status for two disease-modifying antirheumatic drugs each used for 3 months) and with an IMT $>0.9 \mathrm{~mm}$ (Table I). The mean age of the RA patients was $50 \pm 10.4$ years. Our study revealed that most patients had higher Hcy levels. We can see that the Hcy level was normal in only $30 \%$ of patients at baseline. Most of the patients (70\%) had a high level of Hcy. All patients had a high disease activity (DAS-28 score $>5.1$ ) at baseline. The inflammatory markers (ESR and CRP) were highly correlated with the activity of the disease. Most of the patients in our study had a positive rheumatoid factor (RF) (79.13\%). Anti-CCP antibodies were positive in 72 patients (62.60\%). All patients had the presence of one or both of these antibodies (Table I).

After 12 months of anti-TNF- $\alpha$ therapy, patients had a significant decrease in carotid IMT2 values. There were no notable differences between the three groups in regards to the type of anti-TNF- $\alpha$ therapy. There was a strong positive correlation between IMT1-IMT2 in the etanercept group $(\mathrm{P}<0.001, \mathrm{r}=0.758)$, in the adalimumab group $(\mathrm{P}<0.001, \mathrm{r}=0.761)$ and in the infliximab group $(\mathrm{P}<0.001, \mathrm{r}=0.829)$. As well, we observed a strong correlation between the carotid IMT1 and Hcyl levels in RA patients $(\mathrm{P}<0.001)$ at baseline.

The data suggest that plasma Hcy levels and carotid IMT value provide crucial information about the atherosclerotic process and the status of large arteries (carotid artery), even before clinical cardiovascular damage in RA patients. A high Hcyl level at baseline was significantly correlated with carotid IMT2 after 12 months of anti-TNF- $\alpha$ treatment $(\mathrm{P}<0.001, \mathrm{r}=0.463)$. The significant association between Hcy level and IMT is important for the follow-up of the cardiovascular risk in RA patients. After 12 months of treatment, in the 3 groups, IMT2 progression was significantly reduced compared to baseline (Table II). The low level of Hcy2 after 12 months of anti-TNF- $\alpha$ therapy was significantly correlated with a decrease in IMT2 $(\mathrm{P}<0.001)$ in patients who had a high level of Hcy and IMT $>0.9 \mathrm{~mm}$ at baseline. After 12 months of anti-TNF treatment, we observed a decrease in the Hcy level in all the groups $(\mathrm{P}=0.003)$. In the study group, IMT2 was slightly correlated with CRP1 $(\mathrm{P}=0.035)$ and $\mathrm{ESR} 1(\mathrm{P}=0.031)$ serum level at baseline.

The high level of inflammatory markers in RA patients might be augmented by the effect of Hcy level on the progression of carotid atherosclerotic plaque. The association between reduced CRP2 and ESR2 levels and low levels of Hcy2 after anti-TNF therapy can reduce the IMT value in RA patients.

No significant differences were found between the three groups regarding IMT, Hcy level, TC and LDL serum levels, disease activity, and the presence of autoantibodies (RF, anti-CCP) at baseline. DAS-28 values were significantly decreased after 12 months of anti-TNF therapy and were associated with a decrease in carotid IMT2 $(\mathrm{P}<0.001)$. We observed that CRP2 and ESR2 levels were significantly decreased after 12 months of anti-TNF treatment compared to baseline in all groups. In the general group, Hcyl was significantly correlated with CRP1 $(\mathrm{P}<0.008)$ and ESR1 level $(\mathrm{P}=0.003)$ at baseline. After 12 months of anti-TNF treatment, we observed a significant correlation between Hcy2 and the level of CRP1 $(\mathrm{P}=0.001)$ and $\mathrm{ESR} 1(\mathrm{P}=0.001)$.

In addition, the same significant association was observed between Hcyl and IMT1 in the adalimumab group $(\mathrm{P}<0.001)$ 
Table I. Basic characteristics of the RA patients $(\mathrm{N}=115)$.

\begin{tabular}{lc}
\hline Parameter & Values \\
\hline Age (years) & \\
Mean age & $50 \pm 10.4$ \\
$\geq 50$ years & $53 \%(61 / 115)$ \\
$<50$ years & $47 \%(54 / 115)$ \\
Sex (females) & $62.60 \%$ \\
Hcy ( $\mu$ mol/l) & \\
Initial Hcy & $16.36 \pm 9.71$ \\
$\leq 15$ & $30 \%(37 / 115)$ \\
$>15$ & $70 \%(78 / 115)$ \\
CRP (mg/dl) & $35.81 \pm 14.09$ \\
ESR (mm/h) & $34.37 \pm 12.29$ \\
DAS28 & $5 \pm 2.37$ \\
TC (mg/dl) (N=108) & $217.94 \pm 59.40$ \\
LDL (mg/dl) (N=105) & $123.81 \pm 28.35$ \\
IMT (N=115) & \\
Mean IMT mm & $14.43 \pm 31.02$ \\
IMT <0.9 mm & $16.96 \%(19 / 115)$ \\
IMT >0.9 mm & $83.03 \%(96 / 115)$ \\
RF positive & $79.13 \%(91 / 115)$ \\
Anti-CCP positive & $62.60 \%(72 / 115)$ \\
\hline N, the number of patients from whom the analysis was collected; \\
Hcy, homocysteine; CRP, C-reactive protein; ESR, erythrocyte \\
sedimentation rate; DAS-28, Disease Activity Score-28; TC, total \\
cholesterol; LDL, low density lipoprotein; IMT, intima media thick- \\
ness; RF, rheumatoid factor; Anti-CCP, anti-cyclic citrullinated \\
peptide antibodies. \\
\hline
\end{tabular}

and in the infliximab group $(\mathrm{P}<0.001)$. After 12 months of anti TNF therapy, in the general group we observed a significant correlation between Hcy2 and IMT2 $(\mathrm{P}<0.001)$. As we expected, in our study, there was a notable correlation between IMT and CT level at baseline $(\mathrm{P}<0.003)$ and after 12 months $(\mathrm{P}<0.002)$. As well, we observed a significant correlation between IMT1 and LDL1 level $(\mathrm{P}<0.003)$ at baseline. After 12 months of anti TNF therapy IMT2 and LDL2 levels were significantly correlated $(\mathrm{P}<0.002)$. We observed a significant change in the TC levels between baseline and after 12 months. The TC level was decreased after anti-TNF- $\alpha$ therapy. The concomitant treatment with atorvastatin contributed to the decrease in TC and LDL levels, helping reduce the cardiovascular risk in RA patients. At baseline, we found a strong correlation between CT1-IMT1 $(\mathrm{P}<0.001)$ and LDL1-IMT1 $(\mathrm{P}<0.001)$. After 12 months of treatment, the IMT2 value was significantly correlated with $\mathrm{TC} 1$ level $(\mathrm{P}=0.003)$ and LDL1 ( $\mathrm{P}=0.004)$ level. Anti-TNF- $\alpha$ treatment was found to be correlated with a notable increase in both total cholesterol and HDL cholesterol levels and was associated with decreased disease activity. The assessments made after 12 months of anti-TNF- $\alpha$ therapy showed that patients had a significant decrease in carotid IMT2 values. Also, no notable differences were found between the three groups in regards to anti-TNF- $\alpha$ therapy. We did not find a correlation between the level of TC and LDL-cholesterol and the type of disease-modifying antirheumatic drug associated with anti-TNF therapy, at baseline $(\mathrm{P}=0.01)$ and after 12 months $(\mathrm{P}=0.03)$. Patients who received MTX and folic acid associated with biologic therapy had a higher decrease of IMT $(\mathrm{P}=0.001)$ after 12 months than the patients who had associated anti-TNF therapy and leflunomide. It is known that folic acid can induce a decrease in plasma Hcy levels.

\section{Discussion}

We followed the correlation between Hcy level and carotid artery IMT, the two noninvasive indicators for atherosclerosis, in patients with RA under biological treatment. Another aim of the study was to observe the evolution of IMT under treatment, depending on Hcy and other parameters. Hyperhomocysteinemia has been correlated with cardiovascular diseases and several complications as heart attack and stroke (27). Other studies have shown that a high Hcy level is correlated with the development of early atherosclerosis in RA patients. Many studies have demonstrated that hyperhomocysteinemia in RA can result from the disease itself, independent of the treatment used $(27,28)$. In our study, we observed that patients with RA had a high Hcy level. We also observed that a high Hcyl level at baseline was significantly correlated with carotid IMT after 12 months of anti-TNF treatment. The strong association of Hcy levels and IMT is crucial for the follow-up of the cardiovascular risk in RA patients. After 12 months of anti-TNF treatment, the RA patients had a decrease in Hcy level in all the groups, however, this decrease was more significant in the adalimumab group. More studies are necessary to determine if anti-TNF agents have a role in the decrease in Hcy levels in RA patients.

We observed that RA patients who received low-dose MTX associated with folic acid had a more significant decrease in Hcy levels than patients who received leflunomide and this may provide a prevention method against IMT progression. MTX treatment can indirectly increase plasma levels of Hcy in patients with RA, so they must be supplemented with folic acid. It is known that early folic acid supplementation in the first $48 \mathrm{~h}$ after MTX therapy can decrease the high Hcy levels and it should be rational to evaluate Hcy levels within the first $48 \mathrm{~h}$ in patients with RA (29-31).

Other similar studies corroborate our findings. One of these studies showed that IMT can regress after anti-TNF treatment in RA patients (27). This was also observed in our study. A study published in 2012 showed the absence of carotid IMT progression in RA patients treated with anti-TNF therapy (28). Moreover, a similar study conducted on 144 women over 2 years, concluded that anti-TNF agents reduced carotid IMT in RA patients (29). In our study, we found a correlation between inflammatory marker ESR and the IMT value at baseline and after 12 months of TNF- $\alpha$ therapy. Previous studies on high-resolution B-mode ultrasound of the common carotid arteries reported a significant correlation between systemic inflammation markers and the carotid IMT in patients with RA $(18,30)$. Yang et al found correlations between Hcy and CRP, anti-cyclic citrullinated peptide antibodies, RF, and disease activity (DAS-28 score) (29). 
Table II. Patient characteristics according to treatment.

\begin{tabular}{|c|c|c|c|}
\hline Variable & Adalimumab $33 \%(n=38)$ & Etanercept $36.5 \%(n=42)$ & Infliximab $30.5 \%(n=35)$ \\
\hline Men/Women, $\mathrm{n}$ & $16 / 22$ & $14 / 28$ & $13 / 22$ \\
\hline Age (years), mean $\pm \mathrm{SD}$ & $49.79 \pm 10.380$ & $48.31 \pm 10.98$ & $52.97 \pm 9.5$ \\
\hline Hcy1, mean \pm SD & $17.97 \pm 7.24$ & $23.15 \pm 7.88$ & $18.59 \pm 8.17$ \\
\hline Hcy 2, mean \pm SD & $15.37 \pm 4.78$ & $20.16 \pm 6.68$ & $17.12 \pm 7.2$ \\
\hline $\mathrm{IMT} 1$, mean $\pm \mathrm{SD}$ & $1.21 \pm 0.36(\mathrm{n}=35)$ & $1.17 \pm 0.23$ & $1.13 \pm 0.29$ \\
\hline $\mathrm{IMT} 2$, mean $\pm \mathrm{SD}$ & $0.94 \pm 0.25(\mathrm{n}=35)$ & $1.02 \pm 0.21$ & $1.05 \pm 0.23$ \\
\hline $\mathrm{CRP} 1$, mean $\pm \mathrm{SD}$ & $39.74 \pm 13.77$ & $35.06 \pm 16.2$ & $33.34 \pm 9.81$ \\
\hline $\mathrm{CRP} 2$, mean $\pm \mathrm{SD}$ & $4.57 \pm 0.74$ & $4.32 \pm 1.23$ & $4.71 \pm 0.45$ \\
\hline $\mathrm{ESR} 1$, mean $\pm \mathrm{SD}$ & $37.05 \pm 15.08$ & $31.95 \pm 11.66$ & $34.34 \pm 8.92$ \\
\hline $\mathrm{ESR} 2$, mean $\pm \mathrm{SD}$ & $7.03 \pm 2.22$ & $6.74 \pm 2.27$ & $7.03 \pm 2.3$ \\
\hline $\mathrm{TC} 1$, mean $\pm \mathrm{SD}$ & $236.09 \pm 77.07(\mathrm{n}=32)$ & $206.9 \pm 43.92$ & $214.57 \pm 54.72$ \\
\hline $\mathrm{TC} 2$, mean $\pm \mathrm{SD}$ & $162.19 \pm 15.36(n=32)$ & $152.49 \pm 24.71(n=41)$ & $168.57 \pm 14.32$ \\
\hline LDL1, mean \pm SD & $125.13 \pm 33.66(n=32)$ & $124.31 \pm 29.71$ & $122 \pm 22.03$ \\
\hline LDL2, mean \pm SD & $92.81 \pm 5.87(\mathrm{n}=32)$ & $97.44 \pm 16.76(n=39)$ & $92.94 \pm 8.28$ \\
\hline DAS-28_1, mean \pm SD & $6.21 \pm 0.83$ & $5.79 \pm 0.64$ & $6.46 \pm 0.7$ \\
\hline $\begin{array}{l}\text { Anti-CCP antibodies at study } \\
\text { (positive/negative, } n \text { ) }\end{array}$ & $21 / 38$ & $27 / 42$ & $24 / 35$ \\
\hline $\begin{array}{l}\text { RF at study } \\
\text { (positive/negative, } n \text { ) }\end{array}$ & $30 / 38$ & $39 / 42$ & $35 / 35$ \\
\hline \multicolumn{4}{|l|}{ Associated treatment at study } \\
\hline Leflunomide, n (\%) & $16(42.1)$ & $12(28.57)$ & $16(45.71)$ \\
\hline Methotrexate + folic acid, n (\%) & $22(57.9)$ & $30(71.43)$ & $19(54.2)$ \\
\hline
\end{tabular}

1, the first measurement/value; 2, the second measurement/value after 12 months of anti-TNF therapy. Hcy, homocysteine; IMT, intima media thickness; CRP, C-reactive protein; ESR, erythrocyte sedimentation rate; TC, total cholesterol; LDL, low density lipoprotein; DAS28, Disease Activity Score-28; Anti-CCP antibodies, Anti-cyclic citrullinated peptide antibodies; RF, rheumatoid factor; SD, standard deviation.

No notable correlation between CRP levels and IMT was found. In another study, high sensitivity $\mathrm{C}$-reactive protein (hs-CRP), a broadly used marker of inflammation, was not correlated with carotid IMT or the presence of carotid plaques. Hs-CRP has been shown to be correlated with cardiovascular accidents in most studies (32-34). Our results indicate that routine use of hs-CRP may not improve the assessment for early-stage atherosclerosis (35). We observed that the serum concentration of Hcy was increased in RA patients and was associated with high levels of cholesterol and inflammatory markers, an aspect observed in other studies (3). The effect of anti-TNF- $\alpha$ on the lipid profile of patients is controversial. In our study, we detected changes in the profile of TC and LDL. Anti-TNF therapy has been shown to favorably alter lipid profiles with time. Evidence has shown that lipid-lowering medications, statins included, have anti-inflammatory effects $(28,29)$.

Our study had the following limitations: a limited number of patients and a short study period. A larger sample size will give more information on lipid profile, Hcy level, and anti-TNF therapy in RA patients. A long-term follow-up study can provide much more information about carotid IMT and the complications of atherosclerosis in RA patients with anti-TNF therapy. The findings of this study are only suggestive. A longitudinal study with a larger sample size may be helpful to investigate all of the atherosclerosis changes and to evaluate the mortality and morbidity in RA patients.

In conclusion, according to our findings, regardless of the treatment chosen (infliximab, adalimumab, or etanercept), biological treatment reduced the cardiovascular risk in RA. The easiest way to quantify cardiovascular risk is through non-invasive methods, easily accepted by patients and highly reliable. Results from this study support the use of carotid ultrasonography as a fast and easy tool for the prediction of cardiovascular accidents in RA patients. In RA, traditional risk factors can also play a role besides the inflammatory state but alone cannot explain the increased mortality risk in RA patients. The associations between carotid IMT value, Hcy level, and plaque formation are also important for the physicians in the management and follow-up of RA patients who have an increased cardiovascular disease (CVD) risk. Inflammatory markers (CRP, ESR) and Hcy can cause an increase in atherosclerotic cardiovascular disorders through distinct pathways. In RA patients, controlling inflammation alone is not sufficient since there are several factors involved in atherosclerosis such as the Hcy level. Folic acid supplementation should also be included in the treatment of RA patients to prevent methotrexate-related hyperhomocysteinemia. Cardiovascular disease is correlated with high inflammatory 
activity, therefore markers in RA should be investigated. As chronic inflammation is considered a major driver of cardiovascular disease, RA patients should be monitored closely.

\section{Acknowledgements}

Not applicable.

\section{Funding}

No funding was received.

\section{Availability of data and materials}

Any materials or data that are reasonably requested can be provided in a timely fashion to members of the scientific community for noncommercial purposes.

\section{Authors' contributions}

All the authors contributed to the following aspects of this study: Conception and design (mainly DA, EMH, and OGP) and selection, analysis, and interpretation of the cited references (all the authors, but mainly CAS, EMH, OGP, and CFP). Moreover, all the authors were involved in the drafting of the manuscript (mainly CAS, CFP, FIR, and MMN) and in revising it critically for important intellectual content (mainly DA, CAS, CFP, FIR, MMN). All the authors have given their final approval of the version to be published. Thus, all of the authors participated sufficiently in the work and take public responsibility for appropriate portions of the content, so that the authors agree to be accountable for all aspects of the work in ensuring that questions related to the accuracy or integrity of any part of the work are appropriately investigated and resolved.

\section{Ethics approval and consent to participate}

This study was approved by the Research Ethics Committee of 'Dr. Carol Davila' Central Military Emergency University Hospital Bucharest (no. 470). All of the subjects provided written informed consent for publication.

\section{Patient consent for publication}

Not applicable.

\section{Competing interests}

The authors declare that they have no competing interests.

\section{Authors' information}

ORCID numbers: Daniela Anghel 0000-0003-3986-6387; Carmen Adella Sîrbu 0000-0002-1982-1066; Elena-Mădălina Hoinoiu 0000-0001-9016-0822; Oana-Georgiana Petrache 0000-0001-8070-8495; Cristina-Florentina Pleșa 0000-0003-2048-5431; Maria Magdalena Negru 0000-0003-3283-1848; Florentina Ioniță-Radu 0000-0001-9829-5035

\section{References}

1. Essouma M and Noubiap JJ: Therapeutic potential of folic acid supplementation for cardiovascular disease prevention through homocysteine-lowering and blockade in rheumatoid arthritis patients. Biomark Res 3: 24, 2015.

2. Hayta E, Hizmetli S, Atalar MH and Çinar Z: Association of plasma homocysteine level and carotid intima-media thickness in rheumatoid arthritis patients receiving methotrexate. Arch Rheumatol 3: 214-220, 2015.

3. Yang X, Gao F and Liu Y: Association of homocysteine with immunological-inflammatory and metabolic laboratory markers and factors in relation to hyperhomocysteinaemia in rheumatoid arthritis. Clin Exp Rheumatol 33: 900-903, 2015.

4. Snow MH and Mikuls TR: Rheumatoid arthritis and cardiovascular disease: The role of systemic inflammation and evolving strategies of prevention. Curr Opin Rheumatol 17: 234-241, 2005.

5. Pleșa CF, Nicolae C, Sîrbu CA, Nemeș R, Păunescu A and Thânțu MM: Use of anticoagulants in cerebral vascular pathology: Farmacia 67: 27-33, 2019.

6. Maradit-Kremers H, Nicola PJ, Crowson CS, Ballman KV and Gabriel SE: Cardiovascular death in rheumatoid arthritis: A population-based study. Arthritis Rheum 52: 722-732, 2005.

7. Lim TK, Lim E, Dwivedi G, Kooner J and Senior R: Normal value of carotid intima-media thickness-a surrogate marker of atherosclerosis: Quantitative assessment by B-mode carotid ultrasound. J Am Soc Echocardiogr 21: 112-126, 2008.

8. del Rincón I, Williams K, Stern MP, Freeman GL, O'Leary DH and Escalante A: Association between carotid atherosclerosis and markers of inflammation in rheumatoid arthritis patients and healthy subjects. Arthritis Rheum 48: 1833-1840, 2003.

9. Simon A, Gariepy J, Chironi G, Megnien JL and Levenson J: Intima-media thickness: A new tool for diagnosis and treatment of cardiovascular risk. J Hypertens 20: 159-169, 2002.

10. Popa C, Netea MG, van Riel PL, van der Meer JW and Stalenhoef AF: The role of TNF-alpha in chronic inflammatory conditions, intermediary metabolism, and cardiovascular risk. J Lipid Res 48: 751-762, 2007.

11. Linton MF, Yancey PG, Davies SS, Jerome WG, Linton EF, Song WL, Doran AC and Vickers KC: The role of lipids and lipoproteins in atherosclerosis. In: Endotext. Feingold KR, Anawalt B, Boyce A, Chrousos G, de Herder WW, Dhatariya K, Dungan K, Grossman A, Hershman JM, Hofland J, et al (eds). MDText.com, Inc, South Dartmouth, MA, 2019.

12. Giles JT, Post W, Blumenthal RS and Bathon JM: Therapy insight: Managing cardiovascular risk in patients with rheumatoid arthritis. Nat Clin Pract Rheumatol 2: 320-329, 2006.

13. Skeoch S, Cristinacce PLH, Williams H, Pemberton P, Xu D, Sun J, James J, Yuan C, Hatsukami T, Hockings PD, et al: Imaging atherosclerosis in rheumatoid arthritis: Evidence for increased prevalence, altered phenotype and a link between systemic and localised plaque inflammation. Sci Rep 7; 827, 2017.

14. Hadi HA, Carr CS and Al Suwaidi J: Endothelial dysfunction: Cardiovascular risk factors, therapy, and outcome. Vasc Health Risk Manag 3: 183-198, 2005.

15. Saposnik G: The role of vitamin B in stroke prevention: A journey from observational studies to clinical trials and critique of the VITAmins TO Prevent Stroke (VITATOPS). Stroke 42: 838-842, 2011.

16. Selhub J: The many facets of hyperhomocysteinemia: Studies from the Framingham cohorts. J Nutr 136 (Suppl 6): 1726S-1730S: 2006.

17. Alvarez B, Yugueros X, Fernández E, Luccini F, Gené A and Matas M: Relationship between plasma homocysteine and the morphological and immunohistochemical study of carotid plaques in patients with carotid stenosis over 70\%. Ann Vasc Surg 26: 500-505, 2012.

18. Eikelboom JW, Hankey GJ, Anand SS, Lofthouse E, Staples N and Baker RI: Association between high homocyst(e)ine and ischemic stroke due to large- and small-artery disease but not other etiologic subtypes of ischemic stroke. Stroke 5: 1069-1075, 2000.

19. Wu GH, Kong FZ, Dong XF, Wu DF, Guo QZ and Shen AR: Association between hyperhomocysteinemia and stroke with atherosclerosis and small artery occlusion depends on homocysteine metabolism-related vitamin levels in Chinese patients with normal renal function. Metab Brain Dis 3: 859-865, 2017. 
20. Guin A,Chatterjee Adhikari M,Chakraborty S,Sinhamahapatra P and Ghosh A: Effects of disease modifying anti-rheumatic drugs on subclinical atherosclerosis and endothelial dysfunction which has been detected in early rheumatoid arthritis: 1-year follow-up study. Semin Arthritis Rheum 43: 48-54, 2013

21. Kotani K, Miyamoto M and Ando H: The effect of treatments for rheumatoid arthritis on endothelial dysfunction evaluated by flow-mediated vasodilation in patients with rheumatoid arthritis. Curr Vasc Pharmacol 15: 10-18, 2017.

22. Sattin $M$ and Towheed T: The effect of TNF $\alpha$-inhibitors on cardiovascular events in patients with rheumatoid arthritis: An updated systematic review of the literature. Curr Rheumatol Rev 12: 208-222, 2016

23. Popa C, Netea MG, Radstake T, Van der Meer JW, Stalenhoef AF and van Riel PL, Barerra P: Influence of anti-tumour necrosis factor therapy on cardiovascular risk factors in patients with active rheumatoid arthritis. Ann Rheum Dis 2: 303-305, 2005.

24. Dixon WG, Watson KD, Lunt M, Hyrich KL; British Society for Rheumatology Biologics Register Control Centre Consortium, Silman AJ and Symmons DP; British Society for Rheumatology Biologics Register: Reduction in the incidence of myocardial infarction in patients with rheumatoid arthritis who respond to anti-tumor necrosis factor alpha therapy: Results from the British society for rheumatology biologics register. Arthritis Rheum 56: 2905-2912, 2007.

25. Aletaha D, Neogi T, Silman AJ, Funovits J, Felson DT, Bingham CO Jr, Birnbaum NS, Burmester GR, Bykerk VP, Cohen MD, et al: 2010 Rheumatoid arthritis classification criteria: An American college of rheumatology/european league against rheumatism collaborative initiative. Arthritis Rheum 62 : 2569-2581, 2010

26. Kang SS, Wong PW and Malinow MR: Hyperhomocyst(e)inemia as a risk factor for occlusive vascular disease. Annu Rev Nutr 12: 279-298, 1992

27. Roubenoff R, Dellaripa P, Nadeau MR, Abad LW, Muldoon BA, Selhub J and Rosenberg IH: Abnormal homocysteine metabolism in rheumatoid arthritis. Arthritis Rheum 40: 718-722, 1997.
28. Haagsma CJ,Blom HJ, van Riel PL, van't Hof MA, Giesendorf BA, van Oppenraaij-Emmerzaal D and van de Putte LB: Influence of sulphasalazine, methotrexate, and the combination of both on plasma homocysteine concentrations in patients with rheumatoid arthritis. Ann Rheum Dis 58: 79-84, 1999.

29. Yang HT, Lee M, Hong KS, Ovbiagele B and Saver JL: Efficacy of folic acid supplementation in cardiovascular disease prevention: An updated meta-analysis of randomized controlled trials. Eur J Intern Med 23: 745-754, 2012.

30. Gonzalez-Gay MA, Gonzalez-Juanatey C, Piñeiro A Garcia-Porrua C, Testa A and Llorca J: High-grade C-reactive protein elevation correlates with accelerated atherogenesis in patients with rheumatoid arthritis. J Rheumatol 32: 1219-1223, 2005.

31. Morgan SL, Baggott JE, Lee JY and Alarcón GS: Folic acid supplementation prevents deficient blood folate levels and hyperhomocysteinemia during longterm, low dose methotrexate therapy for rheumatoid arthritis: Implications for cardiovascular disease prevention. J Rheumatol 25: 441-446, 1998.

32. Ridker PM, Cushman M, Stampfer MJ, Tracy RP and Hennekens CH: Inflammation, aspirin, and the risk of cardiovascular disease in apparently healthy men. N Engl J Med 336: 973-979, 1997.

33. Ballantyne CM, Hoogeveen RC, Bang H, Coresh J, Folsom AR, Heiss $G$ and Sharrett AR: Lipoprotein-associated phospholipase A2, high-sensitivity C-reactive protein, and risk for incident coronary heart disease in middle-aged men and wome in the atherosclerosis risk in communities (ARIC) study. Circulation 109: 837-842. 2004.

34. Elliott P, Chambers JC, Zhang W, Clarke R, Hopewell JC, Peden JF, Erdmann J, Braund P, Engert JC, Bennett D, et al: Genetic loci associated with C-reactive protein levels and risk of coronary heart disease. JAMA 302: 37-48, 2009.

35. Virani SS, Pompeii L, Lincoln AE, Dunn RE, Tucker AM, Nambi V, Nasir K, Vogel RA, Boone JL, Roberts AJ and Ballantyne CM: Association between traditional cholesterol parameters, lipoprotein particle concentration, novel biomarkers and carotid plaques in retired national football league players. Atherosclerosis 222: 551-556, 2012. 